\title{
EDITORIAL
}

\section{Civil disobedience, climate change and the risks of nuclear accidents}

\author{
Darryl Macer \\ UNESCO Bangkok, 920 Sukhumvit Road, Prakanong, Bangkok 10110, Thailand
}

Should we go beyond our knowledge of an ethical problem to act upon it? The answer to this question may seem, quite obviously, yes! If we do not act, then we cannot expect any ethical problems to be resolved, and the world will not get any better. But how to act? This issue of Ethics in Science and Environmental Politics (ESEP) features an article by John Lemons and Donald A. Brown entitled 'Global climate change and non-violent civil disobedience'. Commentators from different countries and perspectives broadly agree with their conclusions.

The claim is that we cannot expect certain governments to change their concrete actions to reduce greenhouse gas emissions to prevent further climate change because they have not taken sufficient actions over the past decades. Lemons \& Brown (2011, this issue), supported by most commentators, argue that non-violent civil disobedience is a method that could be used to convince governments to act. One suggestion is that consumers start campaigns to boycott products produced by countries who fail to adopt international plans to reduce greenhouse gas emissions, such as the Kyoto Protocol. They also suggest mass public protests and other measures to demand change in climate-change policies.

Lemons \& Brown (2011) focus on the policies of the country they live in, the USA, and particularly criticize the policies of that country. ESEP is an open forum for discussion of ethical issues of science and environmental policies, and cannot condone any targeting of individuals, institutions or countries. It is for the readers to critically assess all points of view, and ESEP will welcome future responses to this and any other paper from all perspectives. In addition, we can see that a number of other countries cannot escape from similar criti- cisms. The commentators in this issue come from a variety of countries, ranging from Mongolia to Europe, and disciplines, including natural science, social science, law, philosophy and sociology. We can see a common framework of discourse being used in a variety of ways.

Industries may also be considered as potential culprits in a similar vein of not adopting policies to mitigate climate change and pollution. In this case, there have been some consumer-led campaigns against particular products. How should consumers be educated about the risks and dangers of energy choices, and lifestyle patterns? The recent naturally induced disaster in Japan that affected several nuclear power plants is a serious challenge for ethics and energy choices. It is an industry that has been backstopped by governments because the insurance risks for the catastrophes that are not meant to happen are too large for private industry. Thus, these nuclear choices are public ones, since public funding is used. At the same time, governments have actively promoted campaigns to claim the safety of nuclear energy. ESEP does not take a particular position on the ethics of nuclear energy; however, we call now for papers in a special issue on the ethics of nuclear energy technology. We invite authors to submit their papers by 31 May 2011, for a rapid publication of a series of papers to critically analyze the ethical issues and context of nuclear energy.

The threats facing biodiversity are numerous, but clearly anthropogenic causes of climate change are one factor in a myriad of activities that adversely affect biodiversity. The Convention on Biological Diversity of 1992 showed that wide-ranging policy on species protection in the international forum is a viable course of action. If we really care about the dramatic loss of bio- 
logical diversity that will limit the choices for future generations, as well as extinguish the lives of entire species on our planet, we need to seriously consider more concrete actions to challenge the decisions of governments.

Academic integrity and sound research and policy analysis are critical pathways towards good decision making for us in the future uses of energy. There are many ways to take action, not just directly limiting our

1The UNESCO Publication 'Energy flow, environment and ethical implications for meat production' can be downloaded from www.unescobkk.org/rushsap/ethics-and-climate-change/ energyethics/eetwg13/ energy use, but changing consumption patterns, shifting away from industrial agriculture, $\underline{1}$ and voting in governments that will prioritise the issues that we value. Research needs to be accompanied by action, and action by research, so I hope that readers will contribute, in future papers and reports, the detailed accounts of the impacts of their actions on government policy, no matter what actions they decide are ethically justified.

\section{LITERATURE CITED}

Lemons J, Brown DA (2011) Global climate change and nonviolent civil disobedience. Ethics Sci Environ Polit 11:3-12 